\title{
A rare pediatric case of left ventricular outflow tract infective endocarditis in hypertrophic cardiomyopathy
}

Madonna E. Lee, MD, ${ }^{a}$ Mariska Kemna, MD, ${ }^{b}$ Amy H. Schultz, MD, ${ }^{b}$ and David Michael McMullan, MD, ${ }^{a}$ Seattle, Wash

From the ${ }^{\mathrm{a} D i v i s i o n}$ of Congenital Cardiac Surgery, Department of Surgery, and ${ }^{\mathrm{b}}$ Division of Cardiology, Seattle Children's Hospital, Seattle, Wash.

Disclosures: The authors reported no conflicts of interest.

The Journal policy requires editors and reviewers to disclose conflicts of interest and to decline handling or reviewing manuscripts for which they may have a conflict of interest. The editors and reviewers of this article have no conflicts of interest.

Received for publication Sept 13, 2020; accepted for publication Sept 17, 2020; available ahead of print Sept 22, 2020.

Address for reprints: Madonna E. Lee, MD, Congenital Cardiac Surgery Department, 4800 Sandpoint Way, NE, RC.2.820, Seattle, Washington 98105 (E-mail: Madonna.lee@ seattlechildrens.org).

JTCVS Techniques 2020;4:281-2

2666-2507

Copyright (c) 2020 The Authors. Published by Elsevier Inc. on behalf of The American Association for Thoracic Surgery. This is an open access article under the CC BY-NC-ND license (http://creativecommons.org/licenses/bync-nd/4.0/).

https://doi.org/10.1016/j.xjtc.2020.09.019

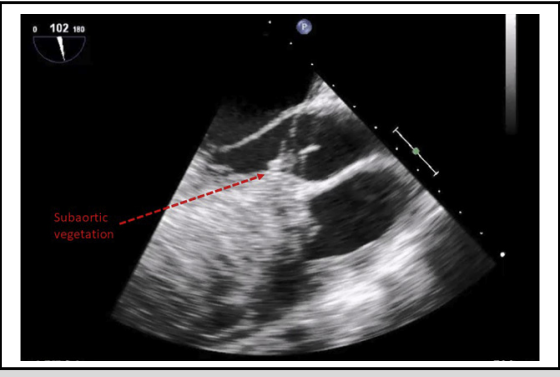

Transesophageal echocardiogram showing severe septal hypertrophy and subaortic endocarditis.

CENTRAL MESSAGE

A rare pediatric presentation of infectious endocarditis in hypertrophic obstructive cardiomyopathy necessitating surgery to address both pathologies.

See Commentaries on pages 283, 285, and 286
Infective endocarditis (IE) is a rare complication in adults with hypertrophic cardiomyopathy (HCM) - incidence of 1.4 to 3.8 per 1000 person-years-most commonly involving the mitral valve. ${ }^{1,2}$ Although infrequent, HCM patients are at increased risk of developing IE with or without left ventricular outflow obstruction (LVOTO). ${ }^{1,3}$ The true incidence of IE is unknown in children with HCM. We report an unusual case of a 15-year-old girl with HCM and severe LVOTO, who developed IE within the LVOT/subaortic region. Due to systemic embolization and persistent fever, she underwent surgical intervention to address both IE and pre-existing LVOTO. Consent to publish was obtained from the patient's parents.

The patient was diagnosed with HCM, trivial aortic insufficiency (AI), and mild LVOTO at age 11 years. Her
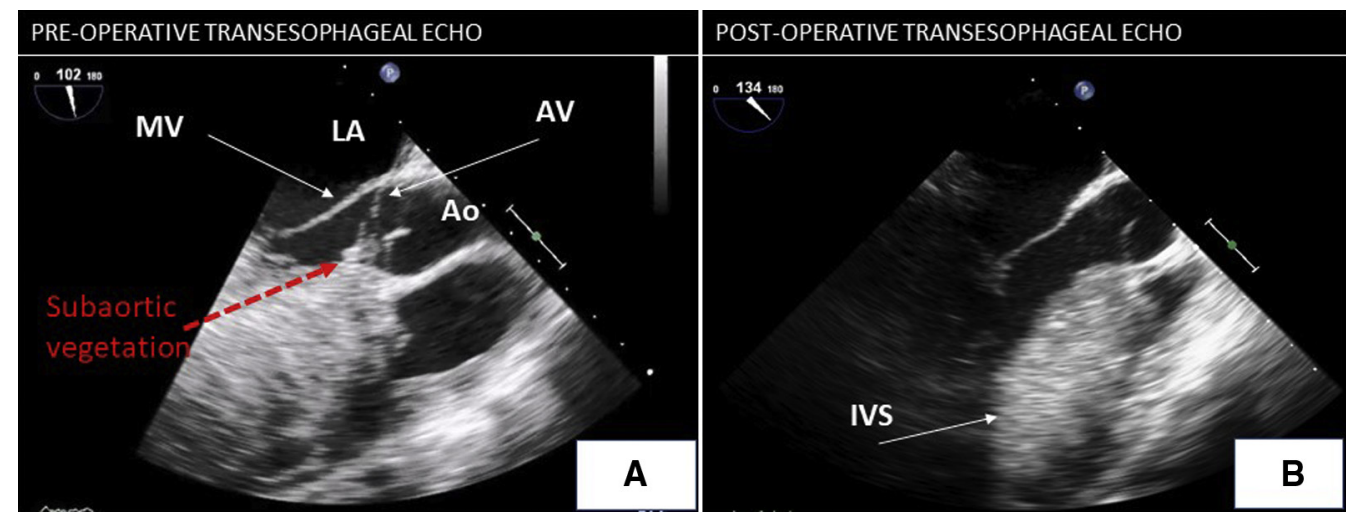

FIGURE 1. Comparison of transesophageal echocardiograph with hypertrophic cardiomyopathy and infectious endocarditis. A, Preoperative showing subaortic vegetation in left ventricular outflow tract (red dotted arrow) and severe septal hypertrophy. B, Postoperative showing excision of vegetation and improved patency of the left ventricular outflow tract. The interventricular septum (white solid arrow) is thickened after septal myectomy. $M V$, Mitral valve; $L A$, left atrium; $A V$, aortic valve; $A o$, aorta; IVS, interventricular septum. 


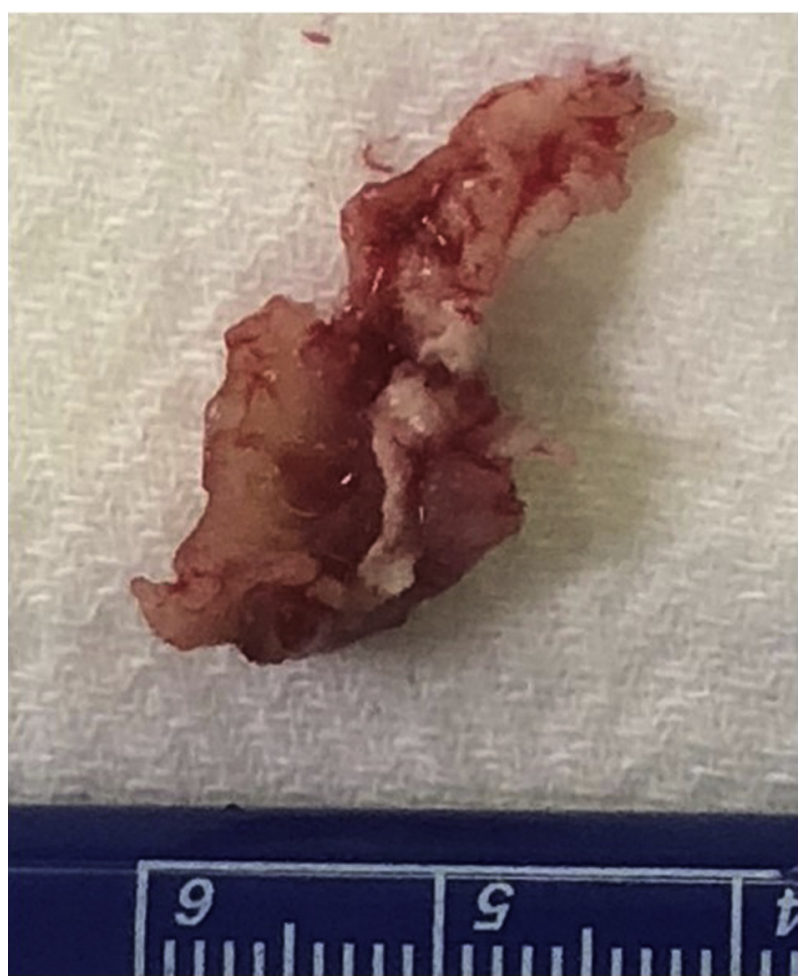

FIGURE 2. Excised, friable subaortic vegetation specimen measuring $1.8 \mathrm{~cm}$ consistent with infectious endocarditis in a patient with concomitant hypertrophic cardiomyopathy.

LVOTO was progressive with peak velocity 4.9 to $5.5 \mathrm{~m} / \mathrm{s}$, peak gradient 96 to $121 \mathrm{~mm} \mathrm{Hg}$, and mild mitral regurgitation (MR). She was prescribed a beta-blocker, and a subcutaneous defibrillator was implanted at age 13 years following an episode of nonsustained ventricular tachycardia. She was admitted after 2 weeks of fever following extensive dental work. She did not receive prophylactic antibiotics per current recommendations. ${ }^{4}$ She was diagnosed with IE based on positive blood cultures (Streptococcus viridans) and echocardiographic evidence of a $1.8-\mathrm{cm}$ crescent-shaped vegetation within the distal LVOT. Despite intravenous antibiotics, she experienced persistent fever and developed Osler's nodes and a splenic infarct. Due to septic emboli and lack of source control, she underwent surgical intervention. Her preoperative transesophageal echocardiogram demonstrated a vegetation, severe LVOTO (peak gradient, $81 \mathrm{~mm} \mathrm{Hg}$; peak velocity; $4.5 \mathrm{~m} / \mathrm{s}$ ), and systolic anterior motion of the mitral valve with moderate MR (Figure 1, A). An extensive septal myectomy was performed, including excision of the vegetation (Figure 2) below the right coronary cusp of the aortic valve via oblique aortotomy. The vegetation did not involve the aortic valve. Postbypass echocardiogram demonstrated no vegetation, but only mildly improved gradient (peak gradient, $49 \mathrm{~mm} \mathrm{Hg}$; peak velocity, $3.5 \mathrm{~m} / \mathrm{s}$ ). Cardiopulmonary bypass was resumed to perform further septal

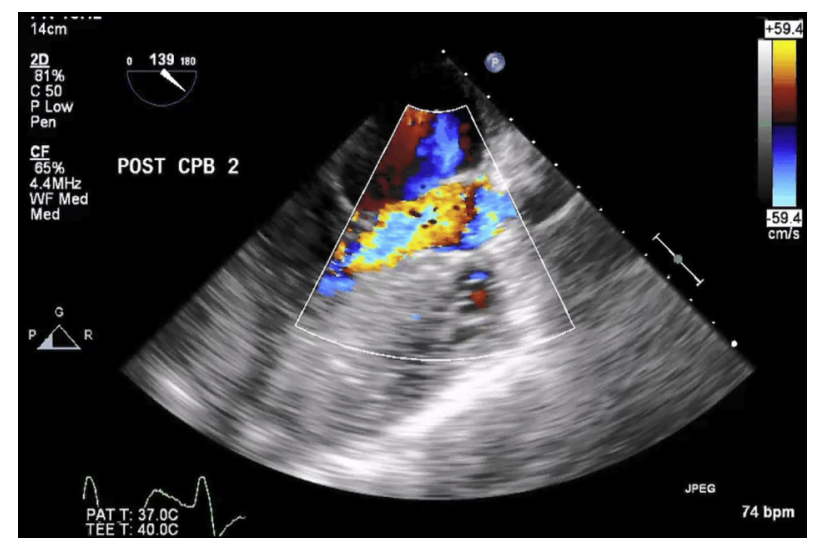

VIDEO 1. A rare pediatric case of infectious endocarditis in hypertrophic obstructive cardiomyopathy. Video available at: https://www.jtcvs.org/ article/S2666-2507(20)30530-7/fulltext.

myectomy. Postbypass transesophageal echocardiogram after the second bypass run demonstrated a significant improvement in LVOTO (peak gradient, $21 \mathrm{~mm} \mathrm{Hg}$; peak velocity, $2.3 \mathrm{~m} / \mathrm{s}$ ), decreased systolic anterior motion, trivial central AI, and trivial MR (Figure 1, B). Despite residual muscle mass, this improvement in LVOT gradient was deemed acceptable because it was less than a threshold peak gradient of $30 \mathrm{~mm} \mathrm{Hg}$.

On postoperative day 10 , she was discharged home on a 6-week course of intravenous antibiotics, with an uneventful recovery. At 3-month follow-up, she is doing well. Postoperative 3-month echocardiograph showed mild acceleration through the LVOT (peak gradient, $23 \mathrm{~mm}$ $\mathrm{Hg}$; peak velocity, $2.4 \mathrm{~m} / \mathrm{s}$ ), trivial-mild AI, and no evidence of recurrent endocarditis.

Although the literature contains case reports and small series describing this rare manifestation of IE in adults with $\mathrm{HCM}^{3-5}$ this case (Video 1) highlights that in the current era pediatric patients with HCM are also at risk for IE and that timely surgical intervention can effectively treat both pathologies simultaneously.

\section{References}

1. Spirito P, Rapezzi C, Bellone P, Betocchi S, Autore C, Conte MR, et al. Infective endocarditis in hypertrophic cardiomyopathy: prevalence, incidence, and indications for antibiotic prophylaxis. Circulation. 1999;99:2132-7.

2. Louahabi T, Drighil A, Habbal R, Azzouzi L. Infective endocarditis complicating hypertrophic obstructive cardiomyopathy. Eur J Echocardiogr. 2006;7:468.

3. Dominguez F, Ramos A, Bouza E, Munoz P, Valerio MC, Farinas MC, et al. Infective endocarditis in hypertrophic cardiomyopathy: a multicenter, prospective, cohort study. Medicine. 2016;95:26.

4. Guler A, Aung SM, Cakal B, Karabay CY, Guler Y, Kirma C. Infective endocarditis complicating hypertrophic obstructive cardiomyopathy: is antibiotic prophylaxis really unnecessary? Curr Cardiol Rev. 2013;9:308-9.

5. Sims JR, Anavekar NS, Bhatia S, O'Horo JC, Geske JB, Chandrasekaran K, et al. Clinical, radiographic, and microbiologic features of infective endocarditis in patients with hypertrophic cardiomyopathy. Am J Cardiol. 2018;121:480-4. 